\title{
Identificación y evaluación de algunos hongos con actividad celulásica aislados en Ecuador
}

\author{
Andrea Rodríguez-Guerra ${ }^{1}$, Charles W. Barnes ${ }^{2}$, María Eugenia Ordóñez ${ }^{1}$, \\ Andrés Salazar ${ }^{1}$ y Carlos A. Soria ${ }^{1}$. \\ ${ }^{1}$ Escuela de Ciencias Biológicas, Pontificia Universidad Católica del Ecuador, Quito; \\ ${ }^{2}$ Centro de Investigación de la Biodiversidad y Cambio Climático, Universidad Tecnológica Indoamérica, Quito. \\ casoria@puce.edu.ec
}

Recibido: 27, 04, 2012; aceptado: 04, 10, 2012

\begin{abstract}
RESUMEN.- Muestras de hongos fueron recolectadas en las estribaciones occidentales y orientales de Ecuador en las provincias de Pichincha, Sucumbíos, Orellana y Santo Domingo de los Tsáchilas. Los hongos fueron asépticamente aislados y multiplicados en Agar Papa Dextrosa (PDA) o Agar Malta (MA). Se observó su crecimiento y luego fueron identificados molecularmente en base a la secuencia de la región ITS1 e ITS2 del ADN ribosomal. Las secuencias obtenidas fueron comparadas con otras similares en Genbank para su análisis filogenético e identificación. Las cepas aisladas e identificadas fueron sembradas en Carboximetilcelulosa (CMC) para evaluar sus capacidades enzimáticas de degradación celulósica. Diámetros (en $\mathrm{mm}$ ) de los halos de hidrólisis fueron continuamente monitoreados durante 7 y 14 días de crecimiento, observándose halos más grandes en las cepas H23.2 y H24 del género Pestalotiopsis y la de L4 del género Neonothopanus con medias significativas. Mayor actividad extracelulásica se registró en los sobrenadantes extraídos de los géneros Neonothopanus, Pestalotiopsis y Gymnopilus a los 14 días de cultivo en el intervalo de $24 \mathrm{~h}$. Esta actividad enzimática en algunos casos no guardó una relación proporcional con la concentración proteica en $\mu \mathrm{g} / \mathrm{ml}$ de los sobrenadantes extraídos de los diferentes cultivos.
\end{abstract}

PALABRAS CLAVES: Celulasas, celulosa, hongos, identificación, proteínas.

ABSTRACT.- Fungal samples were collected in the Western and Eastern Andean foothills of Ecuador in the provinces of Pichincha, Sucumbíos, Orellana and Santo Domingo de los Tsáchilas. The fungi were aseptically isolated and increased on Potato Dextrose Agar (PDA) or Malt Agar (MA). Their growth characteristics were recorded, and molecularly identified on the basis of ribosomal RNA internal transcribed spacer (ITS) region. The sequences were compared with other similar sequences on GenBank for phylogenetic analysis and identification. The isolated and identified strains were plated on Carboxymethylcellulose (CMC) to evaluate their enzymatic capabilities to degrade cellulose. Diameters (in $\mathrm{mm}$ ) of the halos of hydrolysis were continuously monitored during 7-14 days of growth. The halos in strains H23.2 and H24 of the genus Pestalotiopsis and of L4 of the genus Neonothopanus had halo diameters significantly bigger than most of the other fungal samples tested. Increased extracellulolytic activity was recorded in the supernatants extracted from the genera Pestalotiopsis, Neonothopanus, and Gymnopilus after 14 days of culture in the 24-hour interval. This enzyme activity, in some cases, was not proportional to the protein concentration in $\mu \mathrm{g} / \mathrm{ml}$ of the supernatants extracted from the different cultures.

KEYWORDS: Cellulases, cellulose, fungi, identification, proteins. 


\section{INTRODUCCIÓN}

La celulosa es un componente importante de las paredes celulares y probablemente uno de los más abundantes recursos renovables de la naturaleza (Fan et al., 2012). La degradación de la celulosa tiene gran importancia en el ciclo del carbono y son los microorganismos del suelo, hongos y bacterias los que descomponen por hidrólisis esta macromolécula formando varios azúcares (Gefen et al., 2012; Coradetti et al., 2012).

Existen variedades de hongos cuyas enzimas degradan los complejos cristales de celulosa en oligosacáridos, celobiosa o unidades de glucosa (Coradetti et al., 2012; Gaitán y Pérez, 2007). Celulosas y celulasas son utilizadas, por ejemplo, por la industria, en la manufactura de papel, cartón, telas, películas fotográficas, celofanes, diluyentes, jabones, aromas y alimentos (Ponce y Pérez, 2002; Azzaz et al., 2012).

Se han reportado tres tipos de celulasas: la endo B-1,4 glucanasa o carboximetilcelulasa, la exo B-1,4 celobiohidrolasa y la B-1,4 glucosidasa. El nivel de la actividad celulásica y su aplicación depende de cada enzima, de la composición del medio y del proceso de control (Azzaz et al., 2012; Brás et al., 2011).

En la actualidad, la utilización de microorganismos con actividad celulásica representa una solución viable para el problema de la acumulación de residuos celulósicos como resultado de las actividades agrícolas, el desbroce de bosques y la industria en general. El concepto celulásico podría ser una inversión a bajo costo para obtener como resultado una bioremediación aplicable, un sustrato de carbono aprovechable inclusive como biocombustible o la obtención de otros compuestos como algunos productos farmacéuticos, ácidos orgánicos o edulcorantes (Fan et al., 2012; Valencia, 2009; García et al., 2000).

Se han realizado investigaciones sobre el aislamiento, caracterización y producción de variadas enzimas utilizando hongos de los géneros Penicillium, Gymnopilus, Pestalotiopsis o Fusarium (Hongguang et al., 2011; Russell et al., 2011; Qu, 2008; Valentin-Carrera et al., 2008; Jiao, 2006).

No existen estudios acerca de la diversidad de hongos con actividad celulásica en un país mega-diverso como es el caso de Ecuador. El presente trabajo tiene como objetivo principal iniciar estudios moleculares de identificación, evaluación y cuantificación de la capacidad celulásica en medios de cultivo con carboximetilcelulosa utilizando cepas de hongos aislados localmente.

\section{MATERIALES Y MÉTODOS}

Aislamiento.- Muestras de hongos fueron recolectados al azar de materia orgánica en descomposición en las provincias de Pichincha, Sucumbíos, Orellana y Santo Domingo de los Tsáchilas, en Ecuador. Se tomaron muestras de menos de $1 \mathrm{~cm}^{2}$ del estípite del cuerpo fructífero de los hongos recolectados y se sembraron en el campo en medios de Agar Papa Dextrosa (PDA) o Agar Malta (MA) conteniendo estreptomicina al $0.1 \%$ como antibacteriano, $0.2 \%$ de levadura como compuesto de enriquecimiento y $0.01 \%$ de benomil al $50 \%$ como inhibidor de ascomicetos. Estos aislados fueron incubados durante 7 días a $30^{\circ}$ $\mathrm{C}$, al término de los cuales se observó basidiomicetos y un ligero crecimiento de 3 nuevos hongos del grupo de los ascomicetos. De este crecimiento primario, se sembraron todos los aislamientos obtenidos en medios PDA y MA utilizando una cámara de flujo laminar ESCO Optimair ${ }^{\circledR}$. Junto con estos nuevos aislados se realizaron las pruebas preliminares de actividad celulásica cuya metodología se describirá más adelante.

Identificación molecular.- Los cultivos puros obtenidos se multiplicaron y preservaron en PDA sin aditivos. Para la identificación molecular se extrajo ADN del micelio siguiendo un protocolo de extracción con Tiocianato de Guanidina. Se colocó el micelio en tampón de lisis con proteinasa $\mathrm{K}(20 \mathrm{mg} / \mathrm{ml})$ a $55^{\circ} \mathrm{C}$ 
por 3 horas. Las proteínas se precipitaron con una solución 4M de Tiocionato de Guanidina, seguido por la precipitación de ADN con isopropanol. El ADN fue purificado con acetato de sodio $3 \mathrm{M}$ y precipitado nuevamente con etanol al $70 \%$. El ADN concentrado fue almacenado en Tris EDTA pH 8, mientras que las diluciones de ADN usadas para la amplificación se ajustaron a $25 \mathrm{ng} / \mu \mathrm{l}$ en agua destilada estéril. Se amplificó la región ITS1, 5.8S e ITS2 del ADN ribosomal, mediante la reacción en cadena de la polimerasa (PCR) utilizando los cebadores ITS1 e ITS4 (White et al., 1990; Russell et al., 2011). Dos $\mu 1$ de los productos de PCR fueron visualizados en geles de agarosa al $1 \%$ y los $20 \mu 1$ restantes fueron enviados a la compañía Macrogen (Macrogen Inc., Seoul, Korea) para su secuenciación.

Las secuencias obtenidas fueron alineadas con los mejores emparejamientos para cada cepa encontradas con la búsqueda Blastn en GenBank (http://www.ncbi.nlm.nih.gov/ genbank). Se realizó un análisis filogenético de las muestras encontradas y las cepas obtenidas con un método de máxima verosimilitud (PHYML), con 1000 réplicas de bootstrap, utilizando el programa Geneious Pro 5.4.6 (Drummond, 2011).

Descripción morfológica.- La descripción morfológica macroscópica de los cultivos puros de las cepas se efectuó después de cinco días de incubación en MA o PDA. Se levantó una base de datos con fotografías y descripciones de la coloración que presentaba el micelio de cada cepa en ambos medios. Se utilizó la identificación de colores RAL de Pantone (http://www.logorapid.com/tabla_de_colores_ral), tomando en cuenta los colores del centro de la colonia hacia afuera (Logorapid, 2012).

Caracterización del crecimiento y de su actividad celulásica.- Cada cepa de hongo fue sembrada en el centro de cada una de 3 cajas Petri conteniendo $20 \mathrm{ml}$ de Agar Carboxi- metilcelulosa (CMC) al $1 \%$. Las cajas fueron incubadas a $30^{\circ} \mathrm{C}$ en un incubador Incucell de MMM Group durante 2, 4 y 7 días. Al término del tiempo de incubación se procedió a teñir homogéneamente cada cultivo con $5 \mathrm{ml}$ de Rojo Congo al $1 \%$ durante 15 min descartando el tinte sobrante. Se procedió a diluir y lavar el remanente con $5 \mathrm{ml}$ de una solución de $\mathrm{NaCl} 0.1 \mathrm{M}$ durante 15 min eliminándose luego la mayor cantidad del tinte diluido (Gaitán y Pérez, 2007).

Se realizaron 2 mediciones del halo de crecimiento del hongo en cada una de las 3 cajas sembradas utilizando el calibrador digital Tresna ${ }^{\circledR}(0-200 \mathrm{~mm})$ : una medición desde el centro de la colonia hacia el borde exterior del halo, y otra desde el borde interno del halo hacia el exterior del mismo. Posteriormente, se realizó la descripción detallada del tipo de halo observado.

Los datos del tamaño del halo fueron analizados siguiendo un diseño completamente aleatorizado de un factor DCA o Anova 1, con igual tamaño de muestra en el programa SPSS ver. 18, para establecer comparaciones entre la acción enzimática de cada cepa durante los 2 , 4 y 7 días estudiados (Sánchez-Otero, 2011).

Actividad celulásica exoenzimática.Las cepas en estudio fueron cultivadas durante 7 y 14 días a $30^{\circ} \mathrm{C}$ en tubos con $5 \mathrm{ml}$ de una suspensión de CMC al $0.5 \%$ esterilizados a $120^{\circ} \mathrm{C}$ por $15 \mathrm{~min}$ a $1 \mathrm{~atm}$. Los tubos con el cultivo fueron centrifugados durante $20 \mathrm{~min}$ a 2500 rpm (250 g) en una centrífuga Conical Centrifuge Aloe Scientific y se procedió a recuperar el sobrenadante que se guardó a $4^{\circ} \mathrm{C}$ en varios tubos de $1.5 \mathrm{ml}$. Sesenta ul de este sobrenadante fueron incorporados en forma aséptica en un orificio central de $7 \mathrm{~mm}$ de diámetro por $4 \mathrm{~mm}$ de profundidad que se creó con un sacabocados en el Agar CMC al $1 \%$, en cada una de 3 cajas Petri pequeñas $(5 \mathrm{~cm}$ de diámetro, $6 \mathrm{ml}$ de agar) por cepa estudiada.

Los halos de actividad que se formaron a $18^{\circ} \mathrm{C}$ fueron analizados, fotografiados y cuan- 
tificados a las 2, 4, 6, 8 y 24 h después de la inoculación y después de haber sido coloreados con una solución de Rojo Congo similar al procedimiento que se utilizó para visualizar crecimiento y halos de actividad enzimática. Se realizó un Anova multifactorial con diseño aleatorio para las 3 lecturas de cada uno de los 5 intervalos de tiempo $(2,4,6,8,24$ h) en cada una de las 10 cepas incubadas a los 7 y 14 días.

\section{Cuantificación proteica total extrace-} lular por espectrofotometría.- Se analizó la concentración proteica en los sobrenadantes de los cultivos arriba mencionados utilizando el kit de cuantificación de proteínas totales QuantiPro ${ }^{\mathrm{TM}}$ BCA de Sigma con modificaciones implementadas en el laboratorio (Astudillo y Soria, 2011). Los reactivos se prepararon mezclando $500 \mu 1$ de la muestra sobrenadante a analizar con $500 \mu 1$ del reactivo de cuantificación en una proporción 1:1, utilizando tubos de $1.5 \mathrm{ml}$. Los tubos fueron incubados a $60^{\circ} \mathrm{C}$ por aproximadamente 15 min; seguidamente se procedió a medir la absorbancia de la proteína extracelular fúngica a $562 \mathrm{~nm}$ usando el espectrofotómetro Thermo Scientific y a compararla con la absorbancia de las concentraciones estándar de albúmina bovina (BSA) que contenían entre 0 y $500 \mu \mathrm{g}$ de proteína por $\mathrm{ml}$.

Se realizó un Anova factorial con diseño aleatorio para las 2 lecturas de cada una de las absorbancias en las 10 cepas incubadas a los 7 y 14 días. Adicionalmente, se realizó una prueba de Tukey para determinar los subconjuntos homogéneos de los valores de absorbancia en ambos periodos de incubación.

Se realizó además una correlación bivariada de la actividad exoenzimática y de la absorbancia de los extractos de las 10 cepas cultivadas por 7 y 14 días.

\section{RESULTADOS}

Identificación molecular y descripción de los hongos estudiados.- La Tabla 1 describe los datos de colección de los 10 hongos es- tudiados incluyendo su género y/o especie resultante de su identificación molecular en base a secuencias de la región ITS. Los datos de porcentaje de cobertura, máxima identidad y valores de bootstrap para cada cepa se muestran en la Tabla 1. En el caso de las cepas L7 (Psilocybe cubensis), L9 (Agaricus bisporus) y L10 (Agaricus bisporus) donde el porcentaje de cobertura fue igual o mayor a 99 y la identidad máxima fue 100 . No se realizó análisis filogenético, por lo que la Tabla 1 no muestra valores de bootstrap para esas cepas. La cepa L4 (Neothopanus nambi) y la cepa L5 (Gymnopilus junonius) se agruparon con muestras europeas en el análisis filogenético. La cepa L22 fue identificada por su anamorfo, Penicillium pinophilus. En el caso de la cepa L8, la Figura 1 muestra la relación filogenética entre especies de Chloridium según los resultados del Blastn para las secuencias del ITS. La cepa L8 y otra muestreada en la Florida, EEUU, se agrupan como Chloridium virescens aunque difieren notoriamente de otras $C$. virescens igualmente colectadas en ese país.

La Figura 2 muestra la relación filogenética entre especies de Pestalotiopsis según secuencias del ITS con los resultados de Blastn. No se pudo asignar especies para las cepas H23.1, H23.2 y H24 del género Pestalotiopsis ya que no hubo un buen emparejamiento con las secuencias descritas para ese género, inclusive la cepa $\mathrm{H} 24$ resultó diferente a la H23.1 y H23.2.

Características del crecimiento.- La Figura 3 muestra el crecimiento comparativo de cada cepa en PDA y MA; la Tabla 2 describe la coloración del micelio de las mismas. La mayoría de las cepas estudiadas presentan distintos tonos en la coloración según el medio en el que fueron cultivadas pero las características del crecimiento fueron relativamente similares tanto en PDA como en MA para las cepas L4 (N. nambi) y H23.2 (Pestalotiopsis $\mathrm{sp}$.), aunque en algunos casos el PDA favoreció crecimientos más rápidos para L5 $(G$. junonius), L8 (C. virescens), L9 (A. bisporus) 
Tabla 1. Datos de colección e identificación molecular de los hongos estudiados.

\begin{tabular}{|c|c|c|c|c|c|}
\hline $\begin{array}{l}\text { Código } \\
\text { cepa }\end{array}$ & $\begin{array}{l}\text { Especie más } \\
\text { cercanamente } \\
\text { relacionada }^{\mathbf{a}}\end{array}$ & $\begin{array}{l}\text { Localidad } \\
\text { del Blastn }\end{array}$ & $\begin{array}{l}\text { Localidad } \\
\text { Provincia }\end{array}$ & $\begin{array}{l}\text { Número } \\
\text { de } \\
\text { asccesión } \\
\text { id/boot. b }\end{array}$ & $\begin{array}{c}\% \\
\text { cobertura/ } \\
\text { Max. }\end{array}$ \\
\hline L4 & Neonothopanus nambi & Austria & Cuyabeno, Sucumbíos & JX684002 & $86 / 98 / 97$ \\
\hline L5 & Gymnopilus junonius & Francia & Amaguaña, Pinchincha & JX684001 & $100 / 100 / 100$ \\
\hline L7 & Psilocybe cubensis & Varios & Amaguaña, Pichincha & JX684006 & 99/100 \\
\hline L8 & Chloridium virescens $^{c}$ & EEUU & Nanegal, Pichincha & JX684009 & $99 / 100 / 100$ \\
\hline L9 & Agaricus bisporus & Varios & Quito, Pichincha & JX684007 & $100 / 100$ \\
\hline L10 & Agaricus bisporus & Varios & Quito, Pichincha & JX684008 & $100 / 100$ \\
\hline L22 & $\begin{array}{l}\text { Talaromyces } \\
\text { pinophilus (anamorfo: } \\
\text { Penicillium } \\
\text { pinophilus) }\end{array}$ & Varios & El Coca, Orellana & JX684010 & $>99 / 100$ \\
\hline $\mathrm{H} 23.1$ & Pestalotiopsis sp. ${ }^{\mathrm{d}}$ & Varios & $\begin{array}{l}\text { Otonga, Sto } \\
\text { DomTsáchilas }\end{array}$ & JX684004 & $>99 / 100 / 43$ \\
\hline $\mathrm{H} 23.2$ & Pestalotiopsis sp. ${ }^{\mathrm{d}}$ & Varios & $\begin{array}{l}\text { Otonga, Sto } \\
\text { DomTsáchilas }\end{array}$ & JX684005 & $>99 / 100 / 43$ \\
\hline $\mathrm{H} 24$ & Pestalotiopsis sp. $\mathrm{d}$ & Varios & $\begin{array}{l}\text { Otonga, Sto } \\
\text { DomTsáchilas }\end{array}$ & JX684003 & $>99 / 100 / 68$ \\
\hline
\end{tabular}

a. En base a los resultados de Blastn, y del análisis filogenético con especies cercanamente relacionadas.

b. Resultados de Blastn para porcentaje de cobertura e identidad máxima, y valor de bootstrap del último grupo filogenético de la cepa. Se utilizó análisis filogenético cuando los resultados de Blastn no eran obvios.

c. Existen 2 grupos claramente diferentes para Chloridium virescens (ver Figura 2). L8 tiene una secuencia similar solo a una muestra de la Florida, EEUU.

d. El análisis filogenético no mostró buena agrupación con nombres de especies, pero H24 es diferente a H23.1 y H23.2 (ver Figura 1).

y L10 (A. bisporus); mientras que L7 (P. cubensis), L22 (P. pinophilus) y L24 (Pestalotiopsis $\mathrm{sp}$.) crecieron mejor y más rápidamente en MA.

Diferencias y caracterización de la actividad celulásica durante el crecimiento.La cepa que mostró el mayor halo de hidrólisis del CMC a los 7 días de crecida fue $\mathrm{H} 23.2$ Pestalotiopsis sp. (media $=14.63 \mathrm{~mm}$ ), seguida de $\mathrm{H} 24$ (Pestalotiopsis sp.), L4 (N. nambi) y de L9 (A. bisporus) con medias estadísticamente similares de $10.21 \mathrm{~mm}, 9.65 \mathrm{~mm}$ y 8.54 $\mathrm{mm}$, respectivamente.

La prueba de Tukey al 0.05 permitió comparar y confirmar resultados obtenidos de las cepas en estudio. Del análisis de varianza (ANOVA) se obtuvo un coeficiente de varian- za del $14.48 \%$ indicando que no hubo variabilidad aceptable entre los datos experimentales dentro de cada grupo. Existieron diferencias altamente significativas entre los valores de las medias obtenidas para las 10 cepas $(\mathrm{p}=$ 0.000). Considerando la desviación típica, se encontró que no existen variaciones considerables entre las medias de los halos de hidrólisis de las cepas L8 C. virescens $(7.37 \mathrm{~mm})$, L9 A. bisporus, $(8.54 \mathrm{~mm})$, L10 A. bisporus (7.04 mm) y probablemente H23.1 Pestalotiopsis sp. $(6.71 \mathrm{~mm})$. Medias estadísticamente parecidas son las que se observaron en L5 G. junonius $(5.37 \mathrm{~mm}), \mathrm{L} 10$ A. bisporus $(7.04$ $\mathrm{mm})$, L22 P. pinophilus $(5.69 \mathrm{~mm})$ у $\mathrm{H} 23.1$ Pestalotiopsis sp. $(6.71 \mathrm{~mm})$. Se reporta otras similitudes estadísticas para L5 G. junonius (5.37 mm), L7 P. cubensis (4.42 mm), L10 A. 


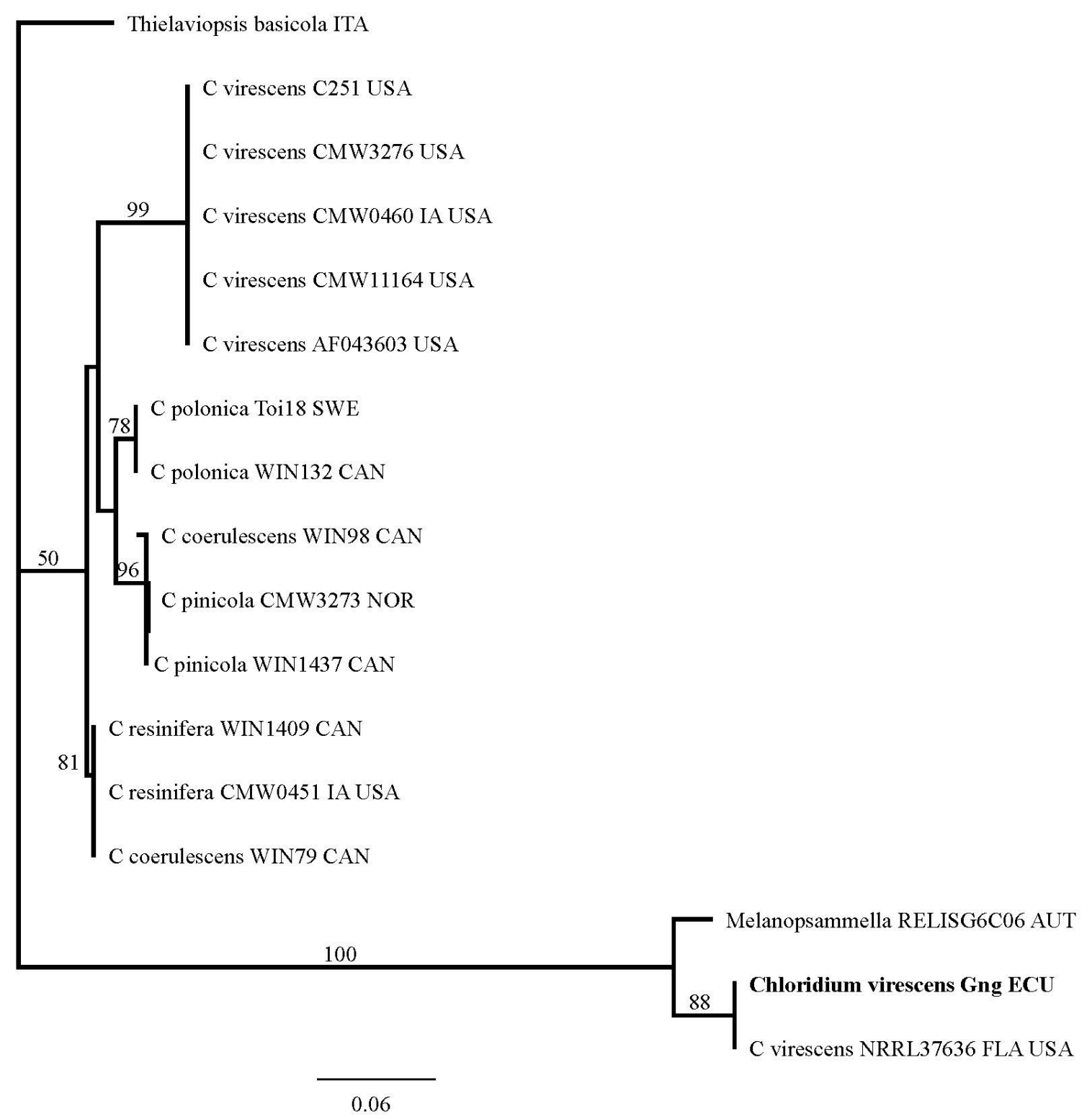

Figura 1. Relación filogenética de las especies de Chloridium según las secuencias de la región ITS del ADNr utilizando PHYLM en Genious. Los números sobre las ramas corresponden a valores de bootstrap $>50 \%$. Se utilizó Thielaviopsis basicola como grupo externo. La muestra en negrilla es la que se utilizó en este estudio. Los nombres de las muestras empiezan con género $(\mathrm{C}=$ Chloridium $)$, código de identificación de la muestra, Estado de los EEUU, y/o código de 3 letras del país.

bisporus $(7.04 \mathrm{~mm})$ y L22 P. pinophilus (5.69 $\mathrm{mm})$, según los datos presentados en la Tabla 3 y en la Figura 4.

Actividad celulásica exoenzimática.- La mayoría de las cepas presentaron halos de hidrólisis del CMC cada vez más grandes a medida que se llegaba al penúltimo ( $8 \mathrm{~h}$ ) y último intervalo $(24 \mathrm{~h})$ del tiempo que duró la prueba $(\mathrm{p}=0.000)$. Las muestras obtenidas de las cepas L4 (N. nambi) y L9 (A. bisporus) cultivadas por 7 días no presentaron actividad durante las 24 h que se estudió la reacción, sin embargo, la actividad enzimática representada por halos con áreas cada vez más grandes a medida que aumentaba el tiempo de reacción hasta las $24 \mathrm{~h}$ fue evidente en los sobrenadantes colectados a 


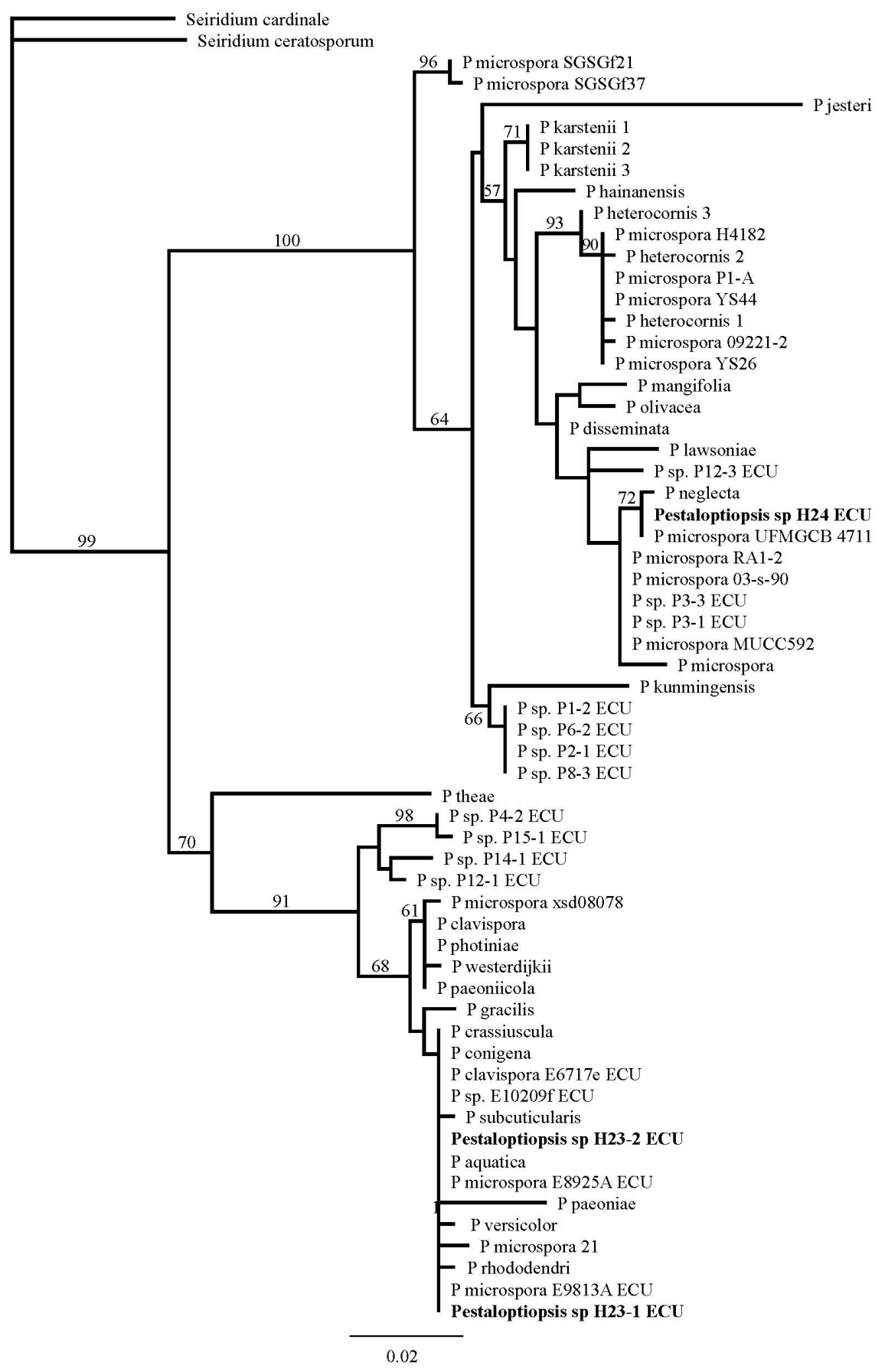

Figura 2. Relación filogenética de las especies de Pestalotiopsis según las secuencias de la región ITS del ADNr utilizando PHYLM en Genious. Los números sobre las ramas corresponden a valores de bootstrap $>50 \%$. Se utilizó a Seiridium cardinale y $S$. ceratosporum como grupos externos. Las muestras que terminan en ECU fueron colectadas en el Ecuador, las muestras en negrillas se utilizaron en este estudio. Los nombres de las muestras empiezan por género $(\mathrm{P}=$ Pestalotiopsis $)$, código de identificación de la muestra, y código de 3 letras del país. 


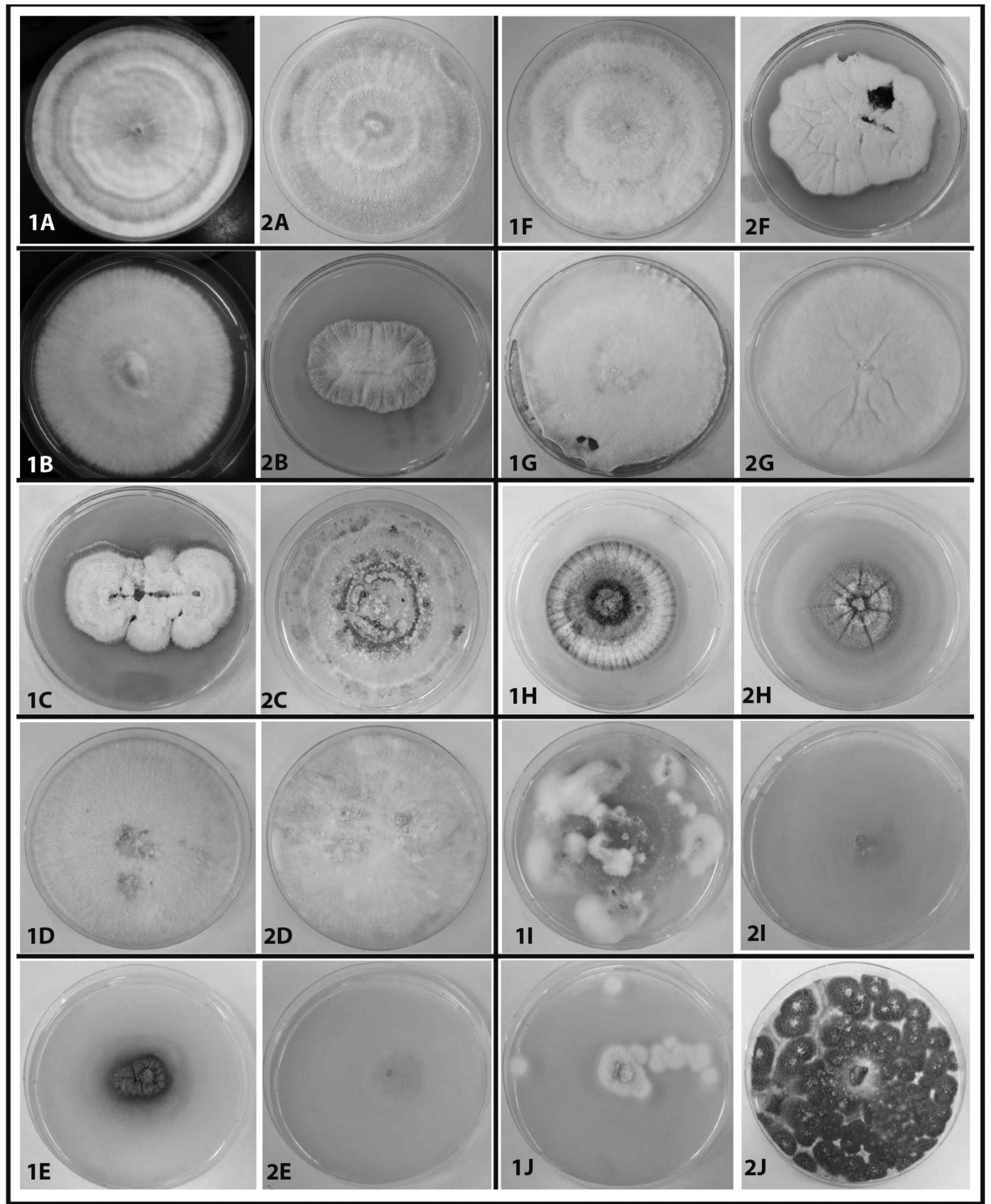

Figura 3. Crecimiento de las cepas en PDA (1) y MA (2): A. Gymnopilus junonius (L5); B. Neonothopanus nambi (L4); C. Pestalotiopsis sp. (H24); D. Psilocybe cubensis (L7); E. Agaricus bisporus (L9); F. Pestalotiopsis sp. (H23.1); G. Pestalotiopsis sp. (H23.2); H. Chloridium virescens (L8); I. Agaricus bisporus (L10); J. Penicillium pinophilus (L22). 
Tabla 2. Crecimiento de las colonias en MA y PDA: descripción de la coloración.

\begin{tabular}{|c|c|c|c|}
\hline \multirow{2}{*}{ Especie } & \multirow{2}{*}{$\begin{array}{l}\text { Código } \\
\text { cepa }\end{array}$} & \multicolumn{2}{|c|}{ Crecimiento } \\
\hline & & En Agar Malta & En Agar PDA \\
\hline $\begin{array}{l}\text { Neonothopanus } \\
\text { nambi }\end{array}$ & L4 & $\begin{array}{l}\text { RAL 1015, RAL 9001, RAL } \\
9002\end{array}$ & RAL 8012, RAL 9001 \\
\hline $\begin{array}{l}\text { Gymnopilus } \\
\text { junonius }\end{array}$ & L5 & $\begin{array}{l}\text { RAL 9001, RAL 9002, RAL } \\
9001\end{array}$ & $\begin{array}{l}\text { RAL 7047, RAL 9003, RAL } \\
\text { 7047, RAL } 9003\end{array}$ \\
\hline $\begin{array}{l}\text { Psilocybe cubensis } \\
\text { Chloridium } \\
\text { virescens }\end{array}$ & $\begin{array}{l}\text { L7 } \\
\text { L8 }\end{array}$ & $\begin{array}{l}\text { RAL 9002, RAL } 9001 \\
\text { RAL 9003, RAL 6028, RAL } \\
\text { 9001, RAL 6028, RAL } 1015\end{array}$ & $\begin{array}{l}\text { RAL } 9001 \\
\text { RAL 6028, RAL 9001, RAL } \\
7044\end{array}$ \\
\hline Agaricus bisporus & L9 & RAL 1023 & RAL 9010, RAL 9001 \\
\hline Agaricus bisporus & L10 & RAL 9001, RAL 1017 & $\begin{array}{l}\text { RAL 8012, RAL 1011, RAL } \\
9001\end{array}$ \\
\hline $\begin{array}{l}\text { Penicillium } \\
\text { pinophilus }\end{array}$ & L22 & $\begin{array}{l}\text { RAL 9001, RAL 6025, RAL } \\
1017\end{array}$ & RAL 9001, RAL 6025 \\
\hline Pestalotiopsis sp. & $\mathrm{H} 23.1$ & RAL 9001 & RAL 9003, RAL 9001 \\
\hline $\begin{array}{l}\text { Pestalotiopsis sp. } \\
\text { Pestalotiopsis sp. }\end{array}$ & $\begin{array}{c}\mathrm{H} 23.2 \\
\mathrm{H} 24\end{array}$ & $\begin{array}{l}\text { RAL 1023, RAL } 9000 \\
\text { RAL 9001, RAL 9003, RAL } \\
\text { 8024, RAL 9001, RAL } 8023\end{array}$ & $\begin{array}{l}\text { RAL } 9001 \\
\text { RAL 9001, RAL 9003, RAL } \\
\text { 8024, RAL 9001, RAL } 8023\end{array}$ \\
\hline
\end{tabular}

los 14 días de cultivo, inclusive mayor a lo detectado en las otras cepas. El comportamiento de $C$. virescens $\mathrm{L} 8$ es diferente porque a las 24 $\mathrm{h}$ de los 7 días de cultivo disminuye su actividad exoenzimática a casi cero.

Se realizó el análisis de varianza de los extractos obtenidos a los 7 y 14 días de cultivo con tres lecturas para cada cepa en cada intervalo de tiempo $(2,4,6,8$ y 24 horas) respectivamente. Los resultados permitieron determinar que los extractos de cultivos obtenidos a los 14 días tuvieron mayor actividad enzimática que los recogidos a los 7 días $(\mathrm{p}=0.000)$. La cepa con mayor actividad enzimática a los 7 días fue la H23.1 (Pestalotiopsis sp.), seguida de H23.2 (Pestalotiopsis sp.) y de H24 (Pestalotiopsis sp.) con medias de $3.78,3.38$ y $3.30 \mathrm{~mm}$ respectivamente, registradas en el intervalo de 24 horas. Con los extractos recogidos en el día 14 del cultivo de las varias cepas, se obtuvieron resultados similares, pero la cepa con mejor actividad celulásica fue la L4 (N. nambi), seguida de la H23.1 (Pestalotiopsis sp.), y L5 (G. junonius) con medias de $3.98,3.59$ y $3.58 \mathrm{~mm}$ respectivamente (Figura 5). El análisis de varianza dio un coeficiente de variación del $28.32 \%$, indicando una ligera variabilidad en los datos del experimento, sin embargo, los resultados presentados se consideran estadísticamente significativos. La prueba de Tukey al 0.05 permitió comparar y separar en rangos de significación las medias obtenidas para cada cepa durante los dos periodos del experimento, 7 y 14 días en conjunto (Tabla 4).

\section{Cuantificación proteica total extracelu-}

lar.- Se determinaron mayores absorbancias, por ende, mayores concentraciones proteicas en las muestras de sobrenadantes colectados a los 14 días de cultivo en comparación con aquellas reportadas a los 7 días. Las medidas de absorbancia obtenidas fueron comparadas y extrapoladas con las absorbancias obtenidas a 562 nm para proteínas estándar de albúmina bovina (BSA) conteniendo entre 0 y $500 \mu \mathrm{g} / \mathrm{ml}$. 
Tabla 3. Valores de las medias en orden descendente, rangos de significación (en superíndices) y desviación típica (en $\mathrm{mm}$ ) de los halos formados después de 7 días de cultivo.

\begin{tabular}{cllc}
\hline Cepa & \multicolumn{1}{c}{ Especie } & Media $(\mathbf{m m})$ & $\begin{array}{c}\text { Desviación } \\
\text { típica } \\
(\mathbf{m m})\end{array}$ \\
\hline H23.2 & Pestalotiopsis sp. & $14.63^{\mathrm{a}}$ & 0.18 \\
H24 & Pestalotiopsis sp. & $10.21^{\mathrm{b}}$ & 1.14 \\
L4 & Neonothopanus nambi & $9.65^{\mathrm{b}, \mathrm{c}}$ & 1.02 \\
L9 & Agaricus bisporus & $8.54^{\mathrm{b}, \mathrm{c}, \mathrm{d}}$ & 1.19 \\
L8 & Chloridium virescens & $7.37^{\mathrm{b}, \mathrm{c}, \mathrm{d}, \mathrm{e}}$ & 0.83 \\
L10 & Agaricus bisporus & $7.04^{\mathrm{b}, \mathrm{d}, \mathrm{d}, \mathrm{e}}$ & 2.01 \\
H23.1 & Pestalotiopsis sp. & $6.71^{\mathrm{c}, \mathrm{d}, \mathrm{e}}$ & 1.09 \\
L22 & Penicillium pinophilus & $5.69^{\mathrm{d}, \mathrm{e}}$ & 0.92 \\
L5 & Gymnopilus junonius & $5.37^{\mathrm{d}, \mathrm{e}}$ & 1.20 \\
L7 & Psilocybe cubensis & $4.42^{\mathrm{e}}$ & 1.15 \\
& & & \\
\hline
\end{tabular}

La muestra extraída a los 7 días de cultivo que presentó la mayor concentración de proteínas totales fue la L10 (A. bisporus) con una absorbancia de $2.31(500 \mu \mathrm{g} / \mathrm{ml})$, seguida de la L9 (A. bisporus) con $2.19(450 \mu \mathrm{g} / \mathrm{ml})$ y L4 (N. nambi) con $2.16(445 \mu \mathrm{g} / \mathrm{ml})$. Las muestras extraídas a los 14 días que presentaron la mayor cantidad de proteínas totales fueron H23.2 (Pestalotiopsis sp.) con 2.84 de absorbancia $(670 \mu \mathrm{g} / \mathrm{ml})$, seguida de L5 (G. junonius) y L9 (A. bisporus) con 2.73 (660 $\mu \mathrm{g} / \mathrm{ml})$ y otras bastante similares como L7 (P. cubensis) con $550 \mu \mathrm{g} / \mathrm{ml}$ y Pestalotiopsis sp. (H24) con $530 \mu \mathrm{g} / \mathrm{ml}$ (Tabla 5). Las cepas cultivadas durante 14 días presentaron mayores concentraciones $(430$ a $660 \mu \mathrm{g} / \mathrm{ml}$ ) de proteínas totales que las cultivadas a los 7 días $(70$ a $500 \mu \mathrm{g} / \mathrm{ml})(\mathrm{p}=0.000)$.

El Anova factorial realizado para las cepas en ambos periodos de tiempo presentó resultados altamente significativos. Las muestras extraídas a los 14 días de cultivo dieron concentraciones de proteínas totales más altas que las encontradas a los 7 días de incubación.

Se comparó los valores de absorbancia obtenidos para los dos periodos de cultivo en conjunto a través de Anova y Tukey al 0.05. Estas pruebas permitieron agrupar los valo- res en subconjuntos (rangos significativos) y se determinó que $C$. virescens (L8) presentó la absorbancia más baja (1.60) seguida de las cepas $N$. nambi (L4) y Pestalotiopsis sp. (H24) con absorbancias entre 2.11 y 2.12 pero similares para ambos cultivos por lo que sus concentraciones se podrían considerar iguales.

Las cepas Pestalotiopsis sp. (H23.2) y (H23.1) desplegaron absorbancias un poco más altas (2.33 y 2.35) pero similares entre ellas, razón por la cual las concentraciones proteicas serían consideradas iguales. Las cepas de G. junonius (L5) y A. bisporus (L9) dieron absorbancias aún mayores (2.42 y 2.46) y bastante similares entre sí. Pero la cepa con mayor absorbancia, por ende, con mayor concentración proteica, fue la L10 (A.bisporus) con un valor promedio de 2.54 (Tabla 6).

La correlación entre la actividad exoenzimática y la absorbancia de los extractos de 7 días no fue significativa ( $p>0.05)$. Por lo tanto no se encuentra relación lineal en los datos mencionados. Sin embargo, la correlación entre la actividad enzimática y concentración proteica de los extractos de 14 días fue significativa, $(p<0.05)$. El gráfico de los datos mostró relación o tendencia inversa, es decir, que a mayor tamaño de halo de acti- 


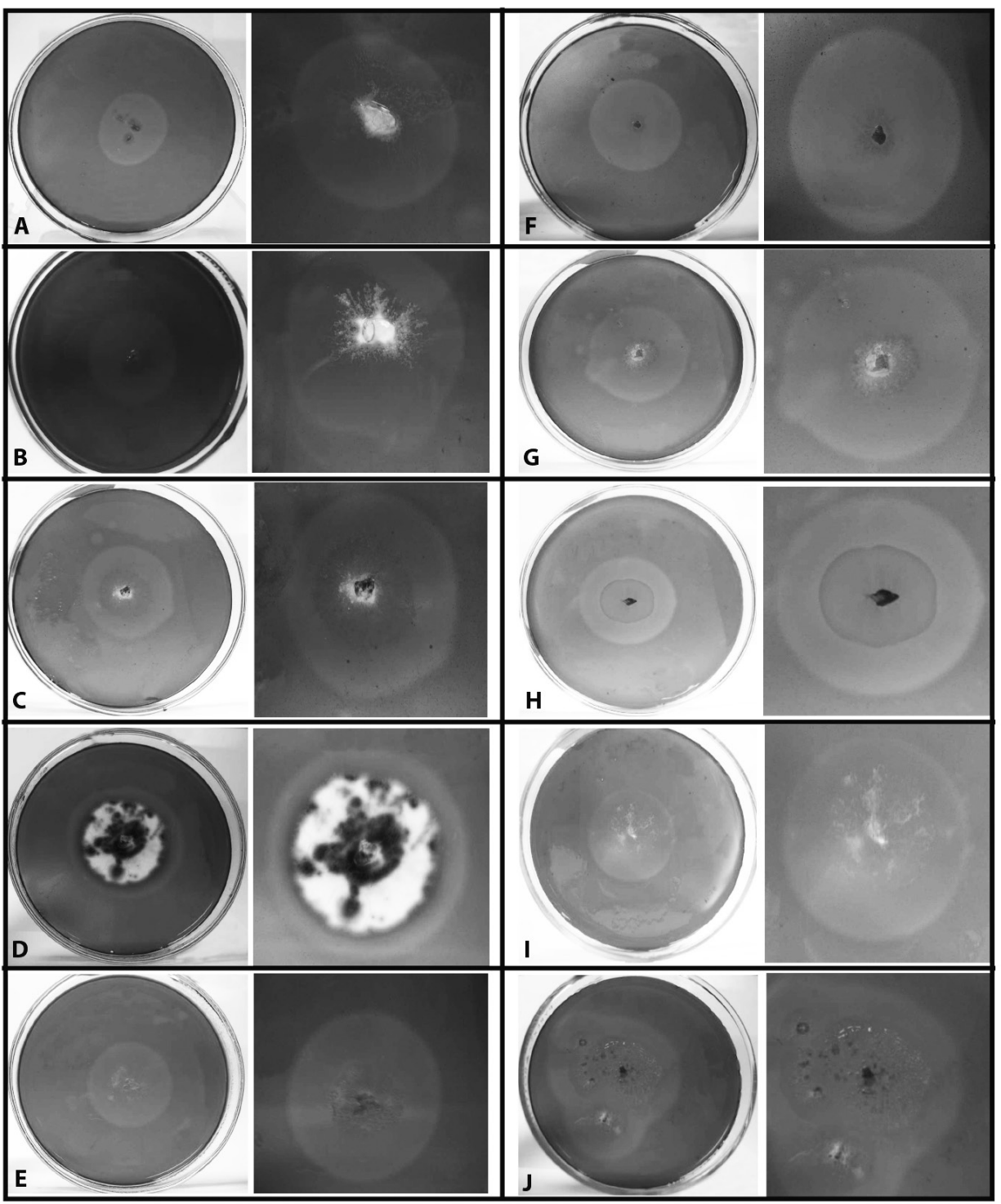

Figura 4. Halos de hidrólisis de celulosa: imágenes normales y de acercamiento para cada cepa cultivada durante 7 días.

A. Gymnopilus iunonius (L5); B. Neonothopanus nambi (L4); C. Pestalotiopsis sp. (H24); D. Psilocybe cubensis (L7); E. Agaricus bisporus (L9); F. Pestalotiopsis sp. (H23.1); G.

Pestalotiopsis sp. (H23.2); H. Chloridium virescens (L8); I. Agaricus bisporus (L10); J. Penicillium pinophilus (L22).

vidad exoenzimática se puede observar en general que las absorbancias registradas son menores lo que indica una menor concentración proteica.

\section{DISCUSIÓN}

Se estudiaron 10 hongos colectados en las estribaciones de la cordillera ecuatoriana. Los hongos fueron aislados, purificados y multiplicados en medios de cultivo MA y PDA, notándose diferencias en cuanto a crecimiento y a coloración en cada medio. El crecimiento tardío de los ascomicetos Chloridium y Pestalotiopsis en medios conteniendo Benomil, pudo haber ocurrido debido a procesos selectivos y de resistencia al fungicida. En general el crecimiento fue relativamente similar en ambos medios, PDA y MA; sin embargo, se observó que PDA permitió un mejor crecimiento en determinadas cepas.

Mediante el análisis de la secuencia de la región ITS1, 5.8S e ITS2 del ADNr, fue posible identificar a todas las cepas estudiadas a nivel de género y a 7 cepas a nivel de especie, obteniéndose valores altos de porcentaje de cobertura, máxima identidad y bootstrap. Fue posible determinar que la cepa de $C$. virescens tiene una relación más cercana con una mues- 

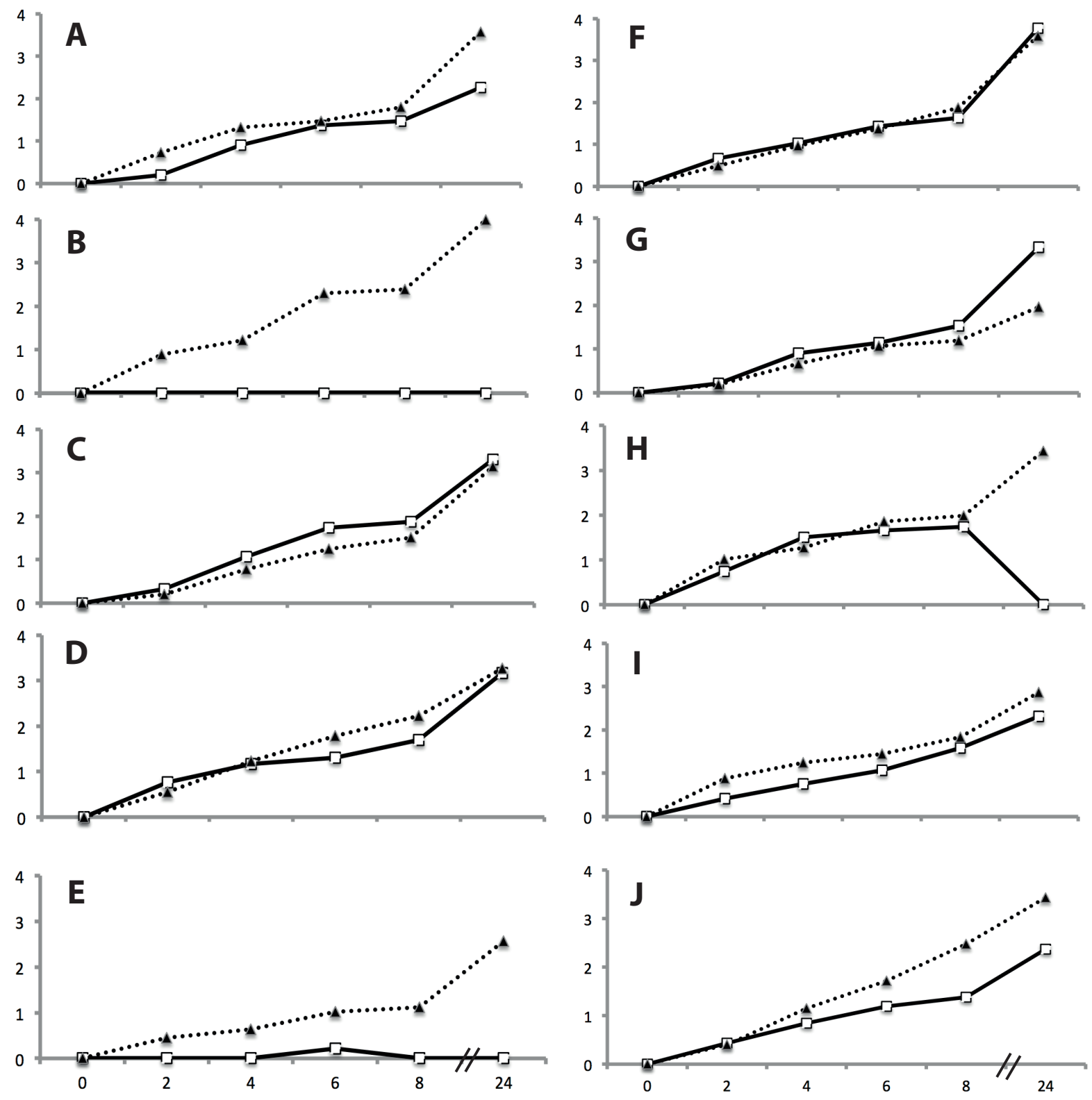

Figura 5. Curvas de actividad enzimática de muestras extraídas a los 7 ( ) y 14 días ( ........ ) de cultivo de las 10 cepas; eje de las y's corresponde a los valores de los halos en mm y el eje de las x's a los intervalos de tiempo de actividad enzimática, 2, 4, 6, 8 y 24 horas: A. Gymnopilus junonius (L5); B. Neonothopanus nambi (L4); C. Pestalotiopsis sp. (H24); D. Psilocybe cubensis (L7); E. Agaricus bisporus (L9); F. Pestalotiopsis sp. (H23.1); G. Pestalotiopsis sp. (H23.2); H. Chloridium virescens (L8); I. Agaricus bisporus (L10); J. Penicillium pinophilus (L22).

tra recolectada en la Florida, EEUU que con otras muestras de la misma especie provenientes de otros lugares de ese país. Probablemente estas dos muestras están más cercanamente relacionadas entre sí por provenir de ambientes tropicales.

Para las cepas del género Pestalotiopsis no fue posible designar la especie ya que el análisis filogenético no mostró una clara agrupación con los nombres de las especies publicadas en GenBank. La designación de especies en este grupo en general no es clara si se basa solo en las secuencias de ITS (Hu et al., 2007), por lo que amerita estudios adicionales para identificar estas cepas hasta un nivel específico.

La Figura 3 registra el crecimiento de los hongos en MA o PDA y la Tabla 2 describe la coloración de dicho crecimiento para cada 
Tabla 4. Valores en orden descendente de las medias y rangos de significación (en superíndices) de los halos formados durante ambos periodos de incubación ( 7 y 14 días).

\begin{tabular}{clc}
\hline Cepa & \multicolumn{1}{c}{ Especie } & Media halos $(\mathbf{m m})$ \\
\hline L7 & Psilocybe cubensis & $1.72^{\mathrm{a}}$ \\
H23.1 & Pestalotiopsis sp. & $1.68^{\mathrm{a}}$ \\
L8 & Chloridium virescens & $1.52^{\mathrm{a}, \mathrm{b}}$ \\
H24 & Pestalotiopsis sp. & $1.52^{\mathrm{a}, \mathrm{b}}$ \\
L22 & Penicillium pinophilus & $1.48^{\mathrm{a}, \mathrm{b}}$ \\
L5 & Gymnopilus junonius & $1.46^{\mathrm{a}, \mathrm{b}}$ \\
L10 & Agaricus bisporus & $1.44^{\mathrm{a}, \mathrm{b}}$ \\
H23.2 & Pestalotiopsis sp. & $1.22^{\mathrm{c}}$ \\
L4 & Neonothopanus & $1.07^{\mathrm{c}}$ \\
L9 & nambi & $0.60^{\mathrm{d}}$ \\
\hline
\end{tabular}

cepa en ambos medios utilizando el identificador de colores RAL de Pantone (http://www. logorapid.com/tabla_de_colores_ral, Logorapid, 2012).

Los resultados indican que hay diferencias notorias entre los crecimientos. Por ejemplo, en A. bisporus (L9) el crecimiento en MA fue lento mientras que en PDA fue más rápido y los bordes del micelio maduro adquieren una consistencia algodonosa de color blanco en PDA y no en MA. Resaltamos también el caso de C. virescens (L8), que al crecer en MA adquiere una coloración blanquecina hacia los bordes del micelio, a diferencia de su crecimiento en PDA que es de color plomizo.

Se encontraron entonces diferencias en la rapidez y en la cantidad de crecimiento, incluso en la coloración que adopta cada hongo en determinado medio. Estas diferencias, en algunos casos muy notorios, podrían hacernos pensar que se trata de cultivos de hongos diferentes, cuando en realidad son morfologías relativamente distintas del mismo individuo y que no pueden ser utilizados como criterios para una identificación final.

La actividad celulásica de estos cultivos fue visualizada y cuantificada en agar CMC teñido con Rojo Congo al término del período indicado de incubación (la concen- tración del CMC fue seleccionada cuidadosamente para favorecer la reacción). El Rojo Congo es una molécula lineal constituida por varios benzenos con radicales azo y aminos que forman puentes de hidrógeno con los hidroxilos de macromoléculas. Estas reacciones permitieron evidenciar o no la hidrólisis de celulosa que se tiñe de rojo cuando reacciona no-covalentemente con el colorante, hecho que no ocurre con las oligocelulosas o unidades de glucosa resultantes de la degradación enzimática. Esta propiedad química facilita la diferenciación entre zonas hidrolizadas o entre organismos celulósicos y no celulósicos (Gaitán y Pérez, 2007). Sería interesante estudiar el comportamiento enzimático o de cultivo en medios como el CMC a diferentes $\mathrm{pH}$ 's, conociendo que muchos hongos crecen mejor y que ciertas enzimas son más activas en condiciones relativamente ácidas.

La endo $\beta$-1,4-glucanasa actúa al azar sobre los enlaces $\beta-1,4$ glucosídicos internos, primero en las regiones amorfas, luego de la parte cristalina de la fibra celulósica, seccionándola en varios conjuntos de $\beta$ oligosacáridos, lo que crea nuevos extremos no reductores que le sirven a otras enzimas como la exo $\beta$-1,4-celobiohidrolasa para remover unidades de celobiosa y a la $\beta-1,4$ glucosidasa para generar glucosa. 
Tabla 5. Absorbancias $(562 \mathrm{~nm})$ y concentración de proteínas totales $(\mu \mathrm{g} / \mathrm{ml})$ promedio de 3 muestras extraídas de cada cepa de hongos cultivados durante 7 y 14 días.

\begin{tabular}{|c|c|c|c|c|}
\hline \multirow[b]{2}{*}{ Especie } & \multicolumn{2}{|c|}{ Siete días de cultivo } & \multicolumn{2}{|c|}{ Catorce días de cultivo } \\
\hline & $\begin{array}{l}\text { Absorbancia } \\
562 \mathrm{~nm}\end{array}$ & $\begin{array}{l}\text { Concentración } \\
\mu \mathrm{g} / \mathrm{ml}\end{array}$ & $\begin{array}{c}\text { Absorbancia } \\
562 \mathrm{~nm}\end{array}$ & $\begin{array}{c}\text { Concentración } \\
\mu \mathrm{g} / \mathrm{ml}\end{array}$ \\
\hline Neonothapanus nambi (L4) & 2.16 & 445 & 2.05 & 430 \\
\hline Gymnopilus junonius (L5) & 2.11 & 440 & 2.73 & 660 \\
\hline Psilocybe cubensis (L7) & 1.97 & 390 & 2.56 & 550 \\
\hline Chloridium virescens (L8) & 0.59 & 70 & 2.61 & 560 \\
\hline Agaricus bisporus (L9) & 2.19 & 450 & 2.73 & 660 \\
\hline Agaricus bisporus (L10) & 2.31 & 500 & 2.76 & 655 \\
\hline Penicillium pinophilus (L22) & 1.87 & 385 & 2.51 & 535 \\
\hline Pestalotiopsis sp. (H23.1) & 2.04 & 420 & 2.67 & 570 \\
\hline Pestalotiopsis sp. (H23.2) & 1.82 & 380 & 2.84 & 670 \\
\hline Pestalotiopsis sp. (H24) & 1.75 & 350 & 2.48 & 530 \\
\hline
\end{tabular}

El acumulamiento tanto de la celobiosa como de la glucosa puede dar lugar a una disminución de la eficiencia enzimática hidrolítica en el cultivo (Ovando-Chacón, 2005; Ryu y Mandels, 1980).

El tamaño del halo de la actividad celulásica permitió comparar estadísticamente la acción enzimática de cada cepa. Según los resultados obtenidos a los 7 días de cultivo se evidenció que los valores promedio de los halos de hidrólisis no presentaron variaciones considerables entre algunas cepas (L8, L10 y H23.1) y (L4, L9 y H24) que se agrupan dentro del mismo rango de variación. Otras cepas como L5, L10, L22 y L23.1 o las cepas L5, L7 y L22 pueden hacer otros grupos, igualmente de acuerdo con sus medias y su rango de variación. Pero la cepa H23.2 es un cultivo que presenta halos bastante grandes y se aleja considerablemente de los demás.

Las cepas con halos grandes (H23.2, H24 y L4) curiosamente tuvieron menor crecimiento del micelio comparado con el resto de cepas que registraron halos más pequeños (entre 4.4 y $8.5 \mathrm{~mm}$ ) y crecimientos similares moderados. Estos resultados nos harían sospechar la presencia de diferencias en la cantidad de exoenzimas producidas por unidad celular, diferencias en la actividad o sinergia por unidad enzimática producida, incluso inhibiciones o estímulos puntuales en la actividad enzimática, dependientes de la acumulación de productos de reacción o metabolitos presentes o ausentes en el medio de cada uno de los cultivos analizados (Gaitán y Pérez, 2007).

La actividad celulásica exoenzimática de los sobrenadantes estudiados fue progresiva en la mayoría de las cepas, observándose un aumento en la generación de exoenzimas durante el periodo de experimentación que fue de 7 o de 14 días de cultivo. El Rojo Congo utilizado para la evaluación de la hidrólisis enzimática, permitió visualizar y cuantificar la capacidad enzimática de degradación del medio de cultivo carboximetilcelulosa. Durante las $24 \mathrm{~h}$ que fueron incubados los extractos exoenzimáticos, las 8 y $24 \mathrm{~h}$ fueron los tiempos que presentaban mayor actividad en ambos sobrenadantes colectados a los 7 y 14 días de cultivo de cada cepa, pero los halos más grandes fueron los que se obtuvieron con muestras colectadas a los 14 días de incubación. La variabilidad registrada en el coeficiente de variación $(\mathrm{CV}=28.32 \%)$ durante el análisis estadístico de la prueba de actividad celulásica pudo haber sido causado por factores que al momento se desconocen, sin embargo, los re- 
Tabla 6. Promedio de los valores de absorbancia de los sobrenadantes extraídos a los 7 y 14 días de cultivo y rangos significativos (en superíndices) obtenidos de la prueba de Tukey.

\begin{tabular}{lcc}
\hline Cepa & Especie & $\begin{array}{c}\text { Media } \\
\text { (absorbancia a } \\
\mathbf{5 6 2} \mathbf{~ n m} \text { ) }\end{array}$ \\
\hline A. bisporus & & $2.54^{\mathrm{a}}$ \\
A. bisporus & $\mathrm{L} 10$ & $2.46^{\mathrm{b}}$ \\
G. junonius & $\mathrm{L} 9$ & $2.42^{\mathrm{b}}$ \\
Pestalotiopsis sp. & $\mathrm{L} 5$ & $2.35^{\mathrm{c}}$ \\
Pestalotiopsis sp. & $\mathrm{H} 23.1$ & $2.33^{\mathrm{c}}$ \\
P. cubensis & $\mathrm{H} 23.2$ & $2.27^{\mathrm{d}}$ \\
P. pinophilus & $\mathrm{L} 7$ & $2.19^{\mathrm{e}}$ \\
Pestalotiopsis sp. & $\mathrm{L} 22$ & $2.12^{\mathrm{e}}$ \\
N. nambi & $\mathrm{H} 24$ & $2.11^{\mathrm{e}}$ \\
C. virescens & $\mathrm{L} 4$ & $1.60^{\mathrm{f}}$ \\
\hline
\end{tabular}

sultados obtenidos fueron considerados como estadísticamente significativos.

Vale la pena también anotar que al comparar las áreas de los halos obtenidos con los sobrenadantes versus los reportados para los cultivos, se encontró que estos últimos fueron mucho más grandes, como es el caso de Pestalotiopsis H23.2 (14.63 mm), Pestalotiopsis $\mathrm{H} 24$ (10.21 mm), N. nambi $(9.65 \mathrm{~mm})$ o A. bisporus L9 $(8.54 \mathrm{~mm})$, comparados con los halos mucho más pequeños de los sobrenadantes (aunque fueron los más grandes del grupo) como el caso de $N$. nambi (3.98 mm), Pestalotiopsis H23.1 (3.78 mm), G. junonius (3.58 $\mathrm{mm})$ o Pestalotiopsis H24 $(3.30 \mathrm{~mm})$. Además el halo no se repite con valores máximos similares para la misma cepa en ambos modelos. Las carboximetilcelulasas del cultivo son mucho más activas que las aisladas del sobrenadante cuya actividad probablemente se ve disminuida por la acumulación de glucosas o celobiosas que en el caso del cultivo son removidas por el crecimiento celular.

Resaltamos también el caso de A. bisporus L9, en el que no se observa actividad exoenzimática en el sobrenadante recogido a los 7 días de cultivo pero si a los 14. La explicación para estos resultados se daría por el hecho de que esta cepa crece muy lentamente en ambos medios de cultivo (Figuras 3, 4 y 5), también porque $A$. bisporus L9 además de tener poco crecimiento y poca actividad enzimática, tiene una concentración alta de proteína en su sobrenadante de 7 días de cultivo (Tabla 4, Figuras 4 y 5), que por lo visto carece de actividad celulásica. No es el caso de A bisporus L10, que además de tener un buen crecimiento y una alta concentración proteica, presenta una actividad enzimática progresiva (Tabla 4, Figuras 4 y 5).

La detección y cuantificación de proteínas con el método BCA se basa en la propiedad de reducir $\mathrm{Cu}^{+2}$ a $\mathrm{Cu}^{+1}$ en medio alcalino y de la habilidad del ácido bicinconínico para quelar $\mathrm{Cu}^{+1}$ y formar complejos hidrosolubles de color que absorben luz a $562 \mathrm{~nm}$, proporcionalmente a la concentración de las proteínas presentes en la muestra (Astudillo y Soria, 2011). Estas reacciones detectaron colorimétricamente y por espectrofotometría la presencia y la cantidad de proteínas totales encontradas en las muestras extraídas de los hongos en estudio.

Los valores proteicos obtenidos son una estimación de la real concentración que depende mucho de la técnica que se utiliza y 
de varios contaminantes orgánicos como carbohidratos, catecolaminas, ácidos nucleicos, lípidos, triptófano, glicerol, cuya presencia afectaría el color, pero la reacción se vuelve confiable una vez estandarizado el $\mathrm{pH}$ de los reactantes, la temperatura y los tiempos de reacción (Zhang et al., 2006).

La concentración proteica encontrada no necesariamente guarda relación lineal con la actividad enzimática de los extractos obtenidos a los 7 días pero la relación es inversamente proporcional con los extraídos a los 14 días; este comportamiento se cree es típico de cada cepa. Es el caso de N. nambi, en el que se encontró máxima actividad exoenzimática $(3.98 \mathrm{~mm})$ en una muy baja concentración proteica $(430 \mu \mathrm{g} / \mathrm{ml})$. Por el contrario, Pestalotiopsis sp. H23.1 y G. junonius L5 mostraron alta actividad enzimática (3.59 y $3.58 \mathrm{~mm}$ ) y concentraciones proteicas igualmente altas $(570$ y $660 \mu \mathrm{g} / \mathrm{ml})$.

Las diferencias en crecimiento, actividad exoenzimática y concentración proteica extracelular son parámetros aparentemente únicos para cada cepa y quizá podrían ser considerados como ayudas en el reconocimiento de géneros y especies que no puedan ser distinguidos con estudios genéticos y de filogenia. Identificar el organismo con el que se trabaja resulta indispensable en estudios de degradación o en aislamientos biomoleculares de interés.

\section{AGRADECIMIENTOS}

A la Pontificia Universidad Católica del Ecuador, por la asignación de fondos de investigación PUCE 2011 y 2012. A la Universidad Tecnológica Indoamérica, por la colaboración en la investigación. Al Ingeniero Julio Sánchez-Otero, por el apoyo estadístico, al Lcdo. Bolívar Salas Mariño, por la recolección de uno de los hongos utilizado en este estudio y a Jorge Flores, por la ayuda en el trabajo de campo y de laboratorio.

\section{REFERENCIAS BIBLIOGRÁFICAS}

Astudillo Y y Soria CA. 2011. Identificación y caracterización de carbohidratos y proteínas en las secreciones del dorso y del pie del molusco Limax flavus. Revista Ecuatoriana de Medicina y Ciencias Biológicas, 32(1 y 2): 33-48.

Azzaz H, Murad HA, Kholif AM, Hanfy MA y Abdel Gawad MH. 2012. Optimization of culture conditions affecting fungal cellulose production. Research Journal of Microbiology, 7(1): 23-31.

Brás JL, Cartmell A, Carvahlo AL, Verzé G, Bayer E, Vazana Y, Correia M, Prates JA, Ratnaparkhe S, Boraston A, Romao M, Fontes C. M y Gilbert H. 2011. Structural insights into a unique cellulose fold and mechanism of cellulose hydrolysis. Proceedings of the National Academy of Sciences, 108(13): 5237-5242.

Coradetti ST, Craig JP, Xiong Y, Shock T, Tian C y Glass L. 2012. Conserved and essential transcription factors for cellulose gene expression in Ascomycete fungi. Proceedings of the National Academy of Sciences, 109(19): 7397-7402.

Drummond AJ, Ashton B, Buxton S, Cheung M, Cooper A, Duran C, Field M, Heled J, Kearse M, Markowitz S, Moir R, Stones-Havas S, Sturrock S, Thierer T y Wilson A. 2011. Geneious v 5.4. Página de Internet: http://www.geneious.com/ Consultada 12-junio-2012.

Fan L, Zhang Z, Yu X, Xue Y y Tan T. 2012. Self-surface assembly of cellulosome with two miniscaffoldins on Saccharomyces cerevisiae for cellulosic ethanol production. Proceedings of the National Academy of Sciences, 109(33): 13260-13265.

Gaitán DM y Pérez LI. 2007. Aislamiento y evaluación de microorganismos celulolíticos a partir de residuos vegetales frescos y en compost generados en un cultivo de Crisantemo (Dendrathema grandiflora). Tesis de Grado para optar al título de Microbiología Industrial. Pontificia Universidad Javeriana, Facultad de Ciencia, Bogotá, Colombia. 
García O, Vidal T, Colom JF, Pastor FJ, Díaz P y Torres AL. 2000. Influencia de la aplicación de celulasas en la fabricación de papel a partir de fibras de eucalipto. Congreso Iberoamericano de investigación en celulosa y papel (CIADICYP). pp. 3.

Gefen G, Anbar M, Morag E, Lamed R y Bayer E. 2012. Enhaced cellulose degradation by targeted integration of cohesion-fused B- glucosidase into the Clostridium thermocellus cellulosome. Proceedings of the National Academy of Sciences, 109(26): 10298-10303.

Hongguang G, Anzhou M, Guohui Z, Juanli Y, Xiu L, Hongxun Z y Guoquiang Z. 2011. Effect of farnesol on Penicillium decumbens's morphology and cellulase production. Peer-reviewed article. BioResources, 6(3): 3252-3259.

$\mathrm{Hu}$ H, Jeewon R, Zhou DQ, Zhou TX y Hyde KD. 2007. Phylogenetic divestity of endophytic Pestalotiopsis species in Pinus armandii and Ribes spp.: evidence from rDNA and B-tubulin gene phylogenies. Fungal Diversity, 24: 1-22.

Jiao P, Swenson DC, Gloer JB y Wicklow DT. 2006. Chloriolide, a twelve-membered macrolide from Chloridium virescens var. chlamydosporum (NRRL 37636). Journal of Natural Products, 69:636-639.

Logorapid, 2012. Tabla de colores RAL, Matadracs S.L. Barcelona, España CIF B-63055651. Página de Internet: http:// www.logorapid.com/tabla_de_colores ral Consultada 1-junio-2012.

Ovando-Chacón. 2005. Preparativos de celulasas comerciales y aplicaciones en procesos extractivos. Universidad y Ciencia, 21(42): 113-122.

Ponce T y Pérez O. 2002. Celulasas y xilanasas en la industria. Revista Avance y Perspectiva. XX Aniversario de Biotecnología y Bioingeniería. 21 (septiembre-octubre): 273-277.
Qu YB. 2008. Cellulolytic enzymes from Penicillium decumbens JU-A10 for biorefinery of lignocellulosics. State Key Laboratory of Microbial Technology, Shandong University, China.

Russell JR, Huang J, Anand P, Kaury K, Sandoval A, Dantzler KW, Hickman D, Jee J, Kimovec FM, Koppstein D, Marks DH, Mittermiller PA, Núñez SJ, Santiago M, Townes MA, Vishnevetsky M, Williams NE, Núñez Vargas MP, Boulanger LA, Bascom-Slack C y Strobel SA. 2011. Biodegradation of Polyester Polyurethano by Endophytic Fungi. Applied and Environmental Microbiology, 77(17): 6076-6084.

Ryu DD y Mandels M. 1980. Cellulases, biosynthesis and applications. Enzyme and Microbial Technology, 2:91-102.

Sánchez-Otero J. 2011. Introducción al Diseño Experimental. Quito, Ecuador, 235 pp. Valencia MF. 2009. Caracterización de cepas de Fusarium aisladas de lesiones de animales, humanos y plantas. Tesis de grado para optar al título de Microbiología industrial. Pontificia Universidad Javeriana, Facultad de Ciencias, Carrera de Microbiología Industrial, Bogotá, Colombia.

Valentin Carrera L, Kabiersch G, Tuomela M, Steffen K y Hatakka A. 2008. Gymnopylus luteofolius - A promising fungus for bioremediation of contaminated sawmill soil. $4^{\text {th }}$ European Bioremediation Conference, ID 129. p. 1-4.

White TJ, Bruns T, Lee S y Taylor JW. 1990. Amplification and direct sequencing of fungal ribosomal RNA genes for phylogenetics. En: PCR Protocols: A Guide to Methods and Applications. Ed. Innis, MA, DH Gelfand: JJ Sninsky and TJ White. Academic Press, Inc., New York, USA. pp. 315-322.

Zhang YH, Himmer ME y Mielenz JR. 2006. Outlook for cellulose improvement: Screening and selection strategies. Biotechnology Advances, 24:452-481. 